obtain occupation from the 1991 census (social destination), as well as geographical location in 1939 and 1991. We examined the movement between three geographical areas (Edinburgh, Glasgow, Other) in Scotland. Four social mobility trajectories were derived. We modelled the relationship between social and geographic mobility and likelihood of having self-reported limiting long term illness (LLTI) at age 65, using logistic regression.

Results Those who were geographically mobile to Edinburgh had the lowest rates of self-reported LLTI and those who remained resident in the Glasgow area had the highest rates. The lowest and highest rates of LLTI were found in the socially-static at the top and bottom of the social scale respectively, with intermediate rates seen in the upwardly and downwardly mobile. However neither social nor spatial mobility were significantly associated with health in later life in the fully adjusted model when highest educational qualifications and cognitive ability were included. Being female, having higher education qualifications and being in a higher social class in childhood and adulthood reduced the likelihood of poor health at age 65 .

Conclusion Although both social class and geographical location were associated with the likelihood of LLTI in later life, social and spatial mobility were not, when factors such as education and cognitive ability were controlled for.

\section{P47 ARE OBESITY AND INFLAMMATION FROM MIDLIFE ASSOCIATED WITH PHYSICAL FATIGABILITY IN OLD AGE? FINDINGS FROM A BRITISH BIRTH COHORT STUDY}

${ }^{1} \mathrm{R}$ Cooper*, ${ }^{1} \mathrm{M}$ Popham, ${ }^{2} \mathrm{AJ}$ Santanasto, ${ }^{2} \mathrm{NW}$ Glynn, ${ }^{1} \mathrm{D}$ Kuh. ${ }^{1} \mathrm{MRC}$ Unit for Lifelong Health and Ageing, UCL, London, UK; ${ }^{2}$ Department of Epidemiology, University of Pittsburgh, Pittsburgh, USA

\subsection{6/jech-2017-SSMAbstracts. 148}

Background Physical fatigability, the level of fatigue experienced while undertaking specified physical tasks, increases with age throughout adulthood. These age-related changes, which reflect reductions in energy availability, precipitate declines in activity participation and function. Higher levels of fatigue are also related to increased risk of disability and premature mortality so it is imperative to identify modifiable risk factors across life associated with physical fatigability. Crosssectional analyses suggest that obesity and inflammation may be associated with increased risk of high physical fatigability. However, whether these inter-related factors act on the same pathway is unclear and requires further investigation in longitudinal studies. We thus aimed to examine the associations of body mass index (BMI) and inflammatory markers from midlife with subsequent levels of perceived physical fatigability in a nationally representative sample.

Methods Up to 2095 men and women from the MRC National Survey of Health and Development, a British cohort followed-up prospectively since birth in 1946, who had valid physical fatigability scores on the Pittsburgh Fatigability Scale (PFS) at age 68 years were included in analyses. Linear regression models were used to assess associations of BMI from age 43 and inflammatory markers (C-reactive protein (CRP) and interleukin-6 (IL-6)) at age 60-64 with continuous PFS scores at age 68. Adjustments were made for sex (where no evidence of interaction was found), long-term limiting illness, physical activity and symptoms of anxiety and depression. All analyses were performed using STATA v14.2.

Results Women had higher mean PFS scores than men (16.0 $(\mathrm{SD}=9.3)$ vs $13.4(\mathrm{SD}=9.0), \mathrm{p}<0.01)$, with higher scores indicating greater perceived physical fatigability. In sex-adjusted analyses, higher BMI and higher levels of CRP and IL-6 were associated with higher PFS scores. For example, participants with $\mathrm{BMI} \geq 30 \mathrm{~kg} / \mathrm{m}^{2}$ at age 43 had sex-adjusted mean PFS scores 4.7 (95\% CI: 3.3-6.1) points higher than those with BMI $20-24.9 \mathrm{~kg} / \mathrm{m}^{2}$ and, those in the highest fifth of IL-6 at age 60-64 had a mean score 4.9 (95\% CI: 3.5-6.3) points higher than those in the bottom fifth. When these associations were mutually adjusted and adjusted for other covariates, higher BMI and IL-6 remained associated with higher PFS scores, whereas associations with CRP were fully attenuated.

Conclusion These findings highlight the potentially important influence of inflammatory and other cardio-metabolic processes on physical fatigability. They suggest that both BMI and inflammation from midlife may be important targets for intervention to reduce the burden of this commonly reported symptom in older populations.

\section{P48 COMPARING BMI WITH SKINFOLDS TO ESTIMATE AGE AT ADIPOSITY REBOUND AND ITS ASSOCIATIONS WITH LATER CARDIO-METABOLIC RISK MARKERS}

${ }^{1} \mathrm{C}$ Di Gravio*, ${ }^{2} \mathrm{GV}$ Krishnaveni, ${ }^{2} \mathrm{R}$ Somashekara, ${ }^{2} \mathrm{SR}$ Veena, ${ }^{2} \mathrm{~K}$ Kumaran, ${ }^{2} \mathrm{M}$ Krishna, ${ }^{2} \mathrm{SC}$ Karat, ${ }^{1} \mathrm{CHD}$ Fall. ${ }^{1} \mathrm{MRC}$ Lifecourse Epidemiology Unit, University of Southampton, Southampton, UK; ${ }^{2}$ Epidemiology Research Unit, CSI Holdsworth Memorial Hospital, Mysore, India

\subsection{6/jech-2017-SSMAbstracts. 149}

Background Adiposity rebound (AR), defined as the lowest point before the second rise in body mass index (BMI), occurs between the ages of 5 and 7. Early age at AR is associated with higher risk of obesity in later life. However, BMI as a measure of adiposity has limitations: first, BMI incorporates both fat and lean mass, second, BMI is calculated from both height and weight. To identify the AR, a direct measure of fat (i.e. skinfold thickness) might be more relevant. We used data from the Mysore Parthenon Birth Cohort to compare relative merits of BMI and skinfolds in identifying $\mathrm{AR}$ and predicting BMI and cardio-metabolic risk factors at 13.5 years.

Methods The cohort was set up in 1997 in Mysore, India, to examine the long-term effects of gestational diabetes on cardiovascular disease risk factors in the offspring. Children were followed-up annually until 5 years, and 6-monthly after that for detailed anthropometry. At 13.5 years, 545 children had measurements of cardio-metabolic risk markers. We used nonlinear splines and regression analyses (STATA version 14) to characterise the subject-specific growth of BMI and skinfolds (sum of triceps and subscapular skinfolds) throughout childhood, and to assess the associations between age at AR, BMI and cardio-metabolic risk factors.

Results BMI and skinfolds had similar trajectories with both reaching their minimum between 5 and 6 years. Average age at AR was similar between the two measures (5.94 years and 5.73 years respectively), with skinfold-derived AR being characterised by higher variability (standard deviation: 1.47 years and 2.18 years respectively). Later age of BMI-derived AR was associated with lower BMI $\left(-0.89 \mathrm{~kg} / \mathrm{m}^{2} ; 95 \% \mathrm{CI}\right.$ : $\left.\left[-1.04,-0.74 \mathrm{~kg} / \mathrm{m}^{2}\right]\right)$, fat mass $(-1.14 \mathrm{~kg} ; 95 \% \mathrm{CI}:[-1.36,-$ $0.91 \mathrm{~kg}])$, HOMA-IR $(-0.12 ; 95 \% \mathrm{CI}:[-0.17,-0.07 \mathrm{~kg}])$ and 